\title{
Transactions of the Royal Society of Tropical Medicine and Hygiene
}

\section{Clinical risk factors for therapeutic failure in kala-azar patients treated with pentavalent antimonials in Nepal}

\author{
S. Rijal ${ }^{\mathrm{a}, *}$, S. Bhandari ${ }^{\mathrm{a}}$, S. Koirala ${ }^{\mathrm{a}}$, R. Singh ${ }^{\mathrm{b}}$, B. Khanal ${ }^{\mathrm{c}}$, L. Loutan ${ }^{\mathrm{d}}$, \\ J.C. Dujardin ${ }^{\mathrm{e}}$, M. Boelaert ${ }^{\mathrm{f}}$, F. Chappuis ${ }^{\mathrm{d}}$ \\ a Department of Internal Medicine, B.P. Koirala Institute of Health Sciences, Dharan 056700, Nepal \\ b Department of Paediatrics, B.P. Koirala Institute of Health Sciences, Dharan 056700, Nepal \\ c Department of Microbiology, B.P. Koirala Institute of Health Sciences, Dharan 056700, Nepal \\ d Division of International and Humanitarian Medicine, Geneva University Hospitals 6, rue Gabrielle-Perret-Gentil, 1211 Geneva 14, Switzerland \\ e Department of Parasitology, Institute of Tropical Medicine, Nationalestraat 155, Antwerp, Belgium \\ ${ }^{\mathrm{f}}$ Department of Public Health, Institute of Tropical Medicine, Nationalestraat 155, Antwerp, Belgium
}

\section{A R T I C L E I N F O}

\section{Article history:}

Received 3 March 2009

Received in revised form 4 August 2009

Accepted 4 August 2009

Available online 1 September 2009

\section{Keywords:}

Kala-azar

Therapeutic failure

Risk factors

Pentavalent antimonials

Control programme

Nepal

\begin{abstract}
A B S T R A C T
Drug-related factors and parasite resistance have been implicated in the failure of pentavalent antimonials $\left(\mathrm{Sb}^{v}\right)$ in the Indian subcontinent; however, little information is available on host-related factors. Parasitologically confirmed kala-azar patients, treatment naïve to $\mathrm{Sb}^{\mathrm{v}}$, were prospectively recruited at a referral hospital in Nepal and were treated under supervision with 30 doses of quality-assured sodium stibogluconate (SSG) $20 \mathrm{mg} / \mathrm{kg} / \mathrm{day}$ and followed for 12 months to assess cure. Analysis of risk factors for treatment failure was assessed in those receiving $\geq 25$ doses and completing 12 months of follow-up. One hundred and ninety-eight cases were treated with SSG and the overall cure rate was $77.3 \%$ (153/198). Of the 181 cases who received $\geq 25$ doses, 12 -month follow-up data were obtained in 169 , comprising 153 patients (90.5\%) with definite cure and 16 (9.5\%) treatment failures. In the final logistic regression model, increased failure to SSG was significantly associated with fever for $\geq 12$ weeks [odds ratio $(\mathrm{OR})=7.4$ ], living in districts bordering the high SSG resistance zone in $\operatorname{Bihar}(\mathrm{OR}=6.1)$, interruption of treatment $(\mathrm{OR}=4.3)$ and ambulatory treatment $(\mathrm{OR}=10.2)$. Early diagnosis and supervised treatment is of paramount importance to prevent treatment failures within the control programme.
\end{abstract}

(c) 2009 Royal Society of Tropical Medicine and Hygiene. Published by Elsevier Ltd. All rights reserved.

\section{Introduction}

Visceral leishmaniasis (VL), or kala-azar, is considered to be one of the most neglected diseases, ${ }^{1}$ with $90 \%$ of the estimated 500000 annual cases occurring in five countries (India, Bangladesh, Sudan, Nepal and Brazil). ${ }^{2}$ Until recently, first-line therapy for VL in all these countries was pentavalent antimonials $\left(\mathrm{Sb}^{\mathrm{v}}\right){ }^{3}$ However, since the

\footnotetext{
* Corresponding author. Tel.: +977 25525 555; fax: +977 25520251

E-mail address: sumanrijal2@yahoo.com (S. Rijal).
}

$1990 \mathrm{~s}$ a sustained and progressive decline in cure rates with $\mathrm{Sb}^{\mathrm{v}}$ has been reported from the Bihar state of India, ${ }^{4,5}$ and indications of similar trends were also observed in Nepal. ${ }^{6}$ In contrast, $\mathrm{Sb}^{\mathrm{v}}$ therapy continues to be effective in HIV-negative kala-azar patients in Bangladesh and East Africa. $^{7-9}$

Several factors have been implicated in the therapeutic failure of $\mathrm{Sb}^{\mathrm{v}}$, either drug-related, such as inadequate or incomplete regimens ${ }^{10}$ or the use of substandard preparations, ${ }^{11}$ or parasite- or host-related. Antimonialresistant parasites have been described among clinical isolates from India and Nepal. ${ }^{12,13}$ 
Beyond HIV status, there is limited information on hostrelated determinants of treatment responsiveness in VL. Leishmania/HIV co-infected patients have low cure rates and almost all patients are known to relapse with any form of treatment. ${ }^{14,15}$ Risk factors for mortality in VL patients have been related to anaemia, severe malnutrition and long duration of illness. ${ }^{16}$ A significant association with treatment failure was also seen in patients presenting with a large spleen at presentation, reflecting more advanced disease. ${ }^{17}$

Recently, new drugs have been proposed as firstline therapy for VL, namely miltefosine ${ }^{18,19}$ and paromomycin. $^{20}$ Also, the use of combination therapy to protect drugs from emerging resistance has been advocated. ${ }^{21}$ There is a need to update therapeutic guidelines for control programmes in disease-endemic countries, while at the same time lessons need to be drawn from the $\mathrm{Sb}^{\mathrm{v}}$ era. Here we report the results of a prospective study on clinical determinants of therapeutic failure with $\mathrm{Sb}^{\mathrm{v}}$ therapy in parasitologically proven kala-azar patients recruited at a tertiary care centre in Nepal.

\section{Materials and methods}

\subsection{Study site and patient recruitment}

This prospective study was conducted at the B.P. Koirala Institute of Health Sciences (BPKIHS), a university hospital in the eastern region of Nepal, from January 2001 to September 2003.

All patients suspected as having VL (fever $\geq 2$ weeks duration with clinical splenomegaly) were admitted for a complete diagnostic work-up. Parasitologically proven kala-azar cases who were treatment-naïve to $\mathrm{Sb}^{\mathrm{v}}$ therapy were enrolled in the study after obtaining informed consent.

\subsection{Study procedures}

Clinicoepidemiological information was recorded on a case record form. Investigations included complete blood count, chemistry, blood culture, urine analysis and chest radiography. HIV serology testing ${ }^{22}$ using two different rapid tests, namely TRI-DOT (J. Mitra \& Co. Pvt. Ltd., New Delhi, India) and Determine (Abbot Japan Co., Tokyo, Japan), was performed only in those patients who failed to respond to sodium stibogluconate (SSG) therapy. Diagnosis of kalaazar was made by Giemsa-stained bone marrow aspirate or spleen aspirate (if bone marrow aspirate was negative) demonstrating Leishman-Donovan bodies, and the parasite density score was determined. ${ }^{23}$

All VL cases were treated with generic SSG (sodium antimony gluconate; Albert David Ltd., Calcutta, India) $20 \mathrm{mg} / \mathrm{kg} /$ day for 30 days. Each batch of SSG was quality checked by the International Dispensary Association (Amsterdam, The Netherlands). Patients were proposed to stay in hospital for the full duration of therapy. However, those who were unable to stay for the full course were discharged after an initial treatment of 5-7 days in the hospital and were provided with extra vials to complete the full course of therapy at the local health facility. A treatment record card was maintained, which also recorded any interruption of therapy. Clinical assessment to define outcome was conducted at the end of therapy and at 3, 6 and 12 months after completion of therapy. Parasitological assessment was repeated at the end of therapy and at 6 months in all patients and at any time during follow-up if relapse was clinically suspected.

Patients were followed up at BPKIHS or were actively traced in their homes if they did not attend BPKIHS on the appointment date. Treatment failure patients were treated with second-line therapy with amphotericin B $1 \mathrm{mg} / \mathrm{kg} /$ day for 14 days.

\subsection{Data analysis}

For the analysis of risk factors for failure of SSG, a per-protocol perspective was adopted, i.e. only kala-azar patients who received $\geq 25$ doses of SSG were included in the analysis. Those who died before Day 25 while being treated with SSG were excluded from the analysis as their death was most likely related to the disease severity with complications such as bleeding or septicaemia. Also, those for whom SSG therapy was switched to the second-line drug amphotericin B owing to side effects were excluded, as were cases that could not be followed up for 12 months after therapy.

\subsection{Case definitions}

Initial cure was defined as a kala-azar case with absence of fever and negative parasitology at the end of SSG therapy. A non-responder was defined as a kala-azar case with positive parasitology after 30 days of SSG therapy. Definite cure was defined as a kala-azar case with negative parasitology and no clinical signs of relapse at 12 months follow-up. A relapse case was defined as a kala-azar case with initial cure but with reappearance of clinical symptoms and signs with positive parasitology during the 12 months followup. SSG treatment failure was defined as a non-responder or relapse case.

\subsection{Statistical procedures}

Univariate analysis was performed for all potential risk factors by computing the odds ratio (OR) and 95\% CI. Pearson's $\chi^{2}$ test was computed and all determinants associated with therapeutic failure at a $P$-value of $<0.2$ were included in the logistic regression. The best model was selected by backward elimination entry. All tests were two-tailed with a significance level of 0.05 . Data were analysed with the STATA software package (StataCorp LP, College Station, TX, USA).

\section{Results}

A total of 301 clinically suspected VL patients presented at BPKIHS during the study period, of which 224 (74.4\%) were parasitologically confirmed kala-azar cases (Figure 1). Eighteen of them had received SSG in the past and eight had contraindications to SSG. Of the remaining 198 patients started on SSG, 181 received $\geq 25$ doses. Of the remaining 


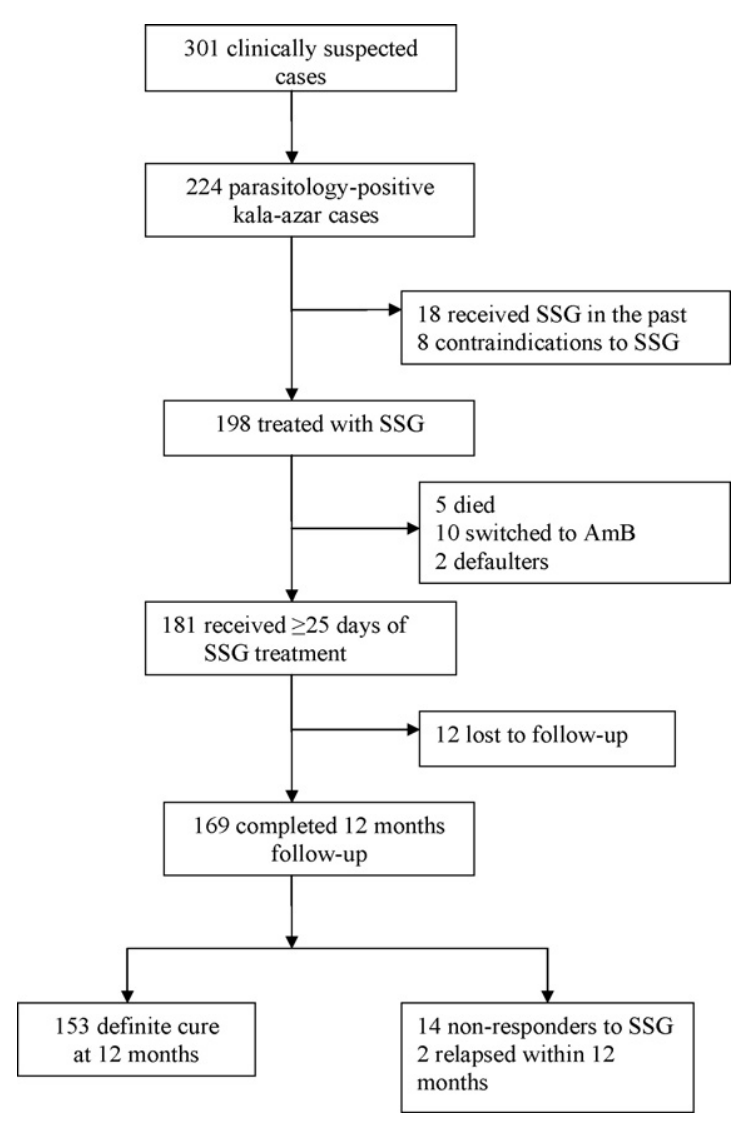

Figure 1. Recruitment and outcome of kala-azar patients. SSG: sodium stibogluconate; AmB: amphotericin B.

17 cases, 5 died during therapy, 2 defaulted and 10 were switched to amphotericin B owing to SSG-related adverse effects. Complete 12-month follow-up was obtained in 169 patients: 153 patients (90.5\%) with definite cure and 16 (9.5\%) treatment failures, which consisted of 14 nonresponses and 2 relapses, the latter at 7 months and 11 months after completion of therapy. Therefore, the overall cure rate on SSG was $77.3 \%$ (153/198), with a $2.5 \%$ death rate (5/198), an $8.1 \%$ failure rate (16/198) and a 5.1\% switch to second-line drug during treatment (10/198), while $6.1 \%$ (12/198) were lost to follow-up.

Risk factor analysis was done on the 169 patients with complete follow-up. The median age in this cohort was 23.9 years (interquartile range $12-35$ years), $44.4 \%$ were female, 164 (97\%) received the full 25 injections at the BPKIHS hospital and $5(3 \%)$ received the injections partly on an outpatient basis. The HIV status was negative in all 16 treatment failures. In the univariate analysis of the association of demographic, clinical and laboratory factors with treatment failure (Supplementary Table 1), there was a strong and significant association of treatment failure with fever of $\geq 12$ weeks duration $(\mathrm{OR}=4.7)$ and with high parasite density (grade $>3)(\mathrm{OR}=3.1)$. Living in a district bordering the high antimony resistance zone in Bihar (district nos. 4, 6 to 12 in Figure 2) and treatment interruption showed a strong association with treatment failure but of borderline statistical significance. The duration of treatment interrup- tion ranged from 3 days to 5 days. Risk factors for SSG failure in the final logistic regression model are shown in Table 1.

\section{Discussion}

This prospective cohort study demonstrated that the cure rate in VL patients treated with SSG in Eastern Nepal was $90.5 \%$ in the per-protocol analysis (i.e. $\geq 25$ daily doses). From an intention-to-treat perspective, SSG cure rates were much lower (77.3\%).

Prolonged fever, treatment interruption, ambulatory treatment and living close to the Bihar high resistance area were independent risk factors for treatment failure with SSG. No other demographic (e.g. age) or clinical (e.g. nutritional status) determinants could be identified. Prolonged fever can be considered as a proxy of disease advancement and in treatment naïve patients implies a long delay in seeking treatment, such has been noted in the subcontinent. ${ }^{7,24}$ Access to VL care in these endemic countries is difficult due to multiple factors: lack of knowledge on VL and health-seeking behaviour in at-risk populations; weakness of the peripheral-level health facilities, with VL care being centralised; and excessive cost of treatment despite free provision of diagnostic tests and drugs by the national Kala-azar Control Programme in Nepal. ${ }^{25}$ With the launch of the VL elimination programme, efforts are being made to decentralise VL care by introducing rapid diagnostic tests (rK39 dipstick) and oral miltefosine therapy at the primary health centre level ${ }^{26}$ as well as adoption of strategies for active case detection. Early diagnosis and treatment would be beneficial both at the individual level by decreasing morbidity and mortality and at the community level by decreasing disease transmission. ${ }^{27}$ Treatment interruption was negatively associated with clinical outcome. In the majority of cases these interruptions were due to running out of available stock of SSG at the hospital level. In India, treatment interruptions have been described with the newer and more expensive drugs such as miltefosine, when sold over the counter, highlighting the need for free and supervised provision of drugs. ${ }^{28}$

Ambulatory treatment, even if directly observed in this study by healthcare providers, was still independently associated with treatment failure. We hypothesise that providers might not always have given the full dose required or may have signed off treatment cards without giving the actual dose. Decentralised treatment of VL should be tightly supervised by the clinicians and programmes, and all patients should be counselled about the importance of treatment adherence, as in DOTS (directly observed treatment short-course) programmes for tuberculosis or antiretroviral therapy for AIDS. This is crucial not only for SSG but also for other antileishmanial treatments (e.g. miltefosine, paromomycin), as widespread inadequate use of drugs has been deeply incriminated in the rise of SSG treatment failure rates in the neighbouring province of Bihar. Strict control of drug delivery and prevention of drug resistance would also be facilitated by the use of short combined treatments such as those currently being studied in India. 29

Increased risk of treatment failure in patients coming from Nepalese districts close to the areas of Bihar where 


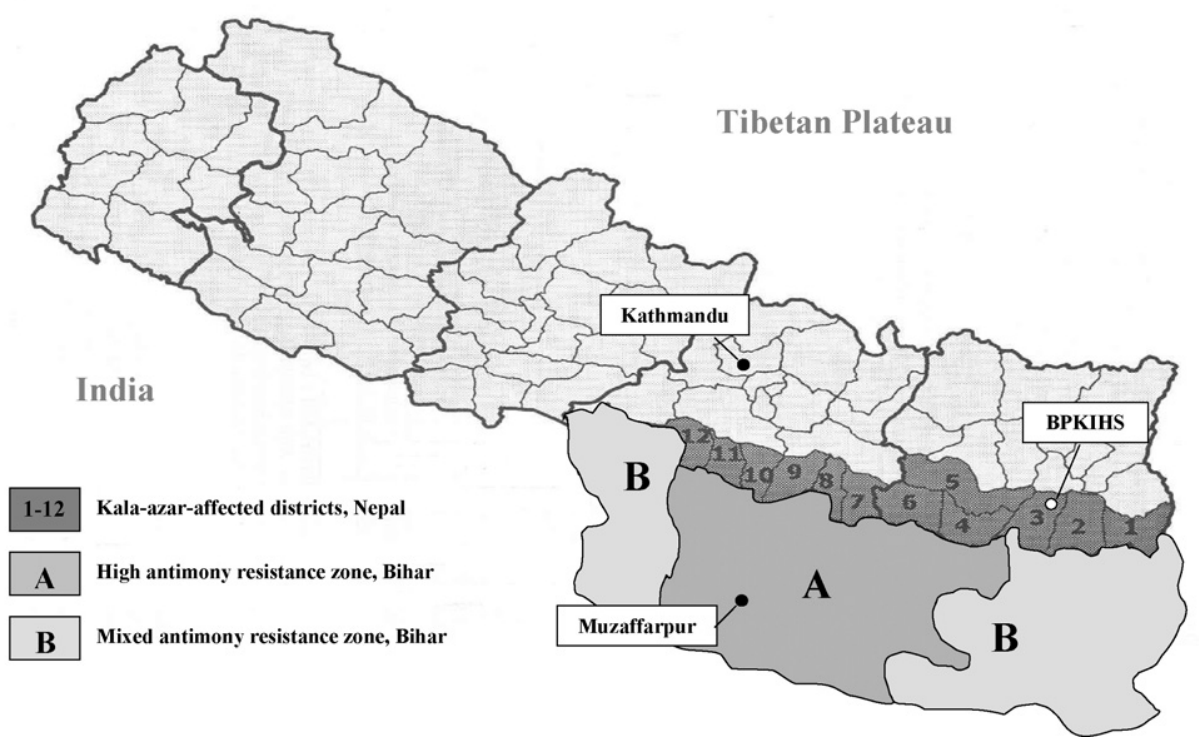

Figure 2. Map of kala-azar-endemic districts in south-eastern Nepal and antimony resistance areas in the state of Bihar, India. BPKIHS: B.P. Koirala Institute of Health Sciences.

Table 1

Final logistic regression model of risk factors for sodium stibogluconate failure in kala-azar patients from eastern Nepal

\begin{tabular}{lrl}
\hline Variable & Odds ratio $(95 \% \mathrm{CI})$ & $P$-value \\
\hline $\begin{array}{l}\text { Districts bordering Bihar high } \\
\text { resistance foci }\end{array}$ & $6.08(1.46-25.34)$ & 0.013 \\
Fever $\geq 12$ weeks & $7.38(2.13-25.59)$ & 0.002 \\
Interruption of treatment & $4.31(1.03-18.06)$ & 0.046 \\
Ambulatory treatment & $10.24(1.25-84.16)$ & 0.03 \\
\hline
\end{tabular}

SSG treatment failure rates exceed 60\% and where resistant strains of Leishmania donovani have been isolated confirms previous findings. 4,6,12 The intense cross-border migration of populations between Nepal and Bihar, considered an important risk factor for the spread of VL in this region, ${ }^{27}$ would also suggest a spread of resistant strains of $L$. donovani from Bihar to Nepal. ${ }^{30}$

Authors' contributions: SR initiated the conception and design of the study and led the writing of the paper; SK and SB were involved in data collection and entry and contributed to the writing of the paper; RS, BK, LL and JCD participated in the conception and design of the paper and contributed to the writing of the paper; FC and MB participated in the conception and design of the paper, data analysis and contributed to the writing of the paper. All authors read and approved the final manuscript. SR is guarantor of the paper.

Acknowledgements: The authors are grateful to Dr Jo Robays from ITM for data analysis, Mr Kamal Parajuli, and the sisters and staff of the Tropical Ward, BPKIHS. They are also grateful to the European Union who supported this project through the Fifth Framework Programme of the European Commission Community Research (contract ICA-CT-2001-10076).
Funding: Fifth Framework Programme of the European Commission Community Research (contract ICA-CT-200110076). The study was conducted and analysed and the results published by the researchers independently of the funding agency.

Conflicts of interest: None declared.

Ethical approval: Ethical Committee of Nepal Health Research Council (Kathmandu, Nepal), September 2000.

\section{Appendix A. Supplementary data}

Supplementary data associated with this article can be found, in the online version, at doi:10.1016/j.trstmh.2009.08.002.

\section{References}

1. Yamey G, Torreele E. The world's most neglected diseases. BMJ 2002;325:176-7. Erratum. BMJ 2002;325:352.

2. WHO. The leishmaniases and Leishmania/HIV co-infections. Geneva: World Health Organization; 2000. Fact sheet No. 116. http://www.who.int/mediacentre/factsheets/fs116/en/print.html [accessed 25 February 2009].

3. WHO. Manual on visceral leishmaniasis control. Geneva: World Health Organization; 1996. WHO/LEISH/96.40.

4. Sundar S. Drug resistance in Indian visceral leishmaniasis. Trop Med Int Health 2001;6:849-54.

5. Sundar S, More DK, Singh MK, Singh VP, Sharma S, Makharia A, et al. Failure of pentavalent antimony in visceral leishmaniasis in India: report from the center of the Indian epidemic. Clin Infect Dis 2000;31:1104-7.

6. Rijal S, Chappuis F, Singh R, Bovier PA, Acharya P, Karki BM, et al. Treatment of visceral leishmaniasis in south-eastern Nepal: decreasing efficacy of sodium stibogluconate and need for a policy to limit further decline. Trans R Soc Trop Med Hyg 2003;97:350-4.

7. Anoopa SD, Bern C, Varghese B, Chowdhury R, Haque R, Ali M, et al. The economic impact of visceral leishmaniasis on households in Bangladesh. Trop Med Int Health 2006;11:757-64.

8. Veeken H, Ritmeijer K, Seaman J, Davidson R. A randomized comparison of branded sodium stibogluconate and generic sodium 
stibogluconate for the treatment of visceral leishmaniasis under field conditions in Sudan. Trop Med Int Health 2000;5:312-7.

9. Ritmeijer K, Dejenie A, Assefa Y, Hundie TB, Mesure J, Boots G, et al. A comparison of miltefosine and sodium stibogluconate for treatment of visceral leishmaniasis in an Ethiopian population with high prevalence of HIV infection. Clin Infect Dis 2006;43: 357-64.

10. Sundar S, Thakur BB, Tandon AK, Agrawal NR, Mishra CP, Mahapatra TM, et al. Clinicoepidemiological study of drug resistance in Indian kala-azar. BMJ 1994;308:307.

11. Rijal S, Chappuis F, Singh R, Boelaert M, Loutan L, Koirala S. Sodium stibogluconate cardiotoxicity and safety of generics. Trans $R$ Soc Trop Med Hyg 2003;97:597-8.

12. Lira R, Sundar S, Makharia A, Kenney R, Gam A, Saraiva E, et al. Evidence that the high incidence of treatment failures in Indian kala-azar is due to the emergence of antimony-resistant strains of Leishmania donovani.J Infect Dis 1999;180:564-7.

13. Rijal S, Yardley V, Chappuis F, Decuypere S, Khanal B, Singh R, et al. Antimonial treatment of visceral leishmaniasis: are current in vitro susceptibility assays adequate for prognosis of in vivo therapy outcome? Microbes Infect 2007;9:529-35.

14. Laguna F, Lopez-Velez R, Pulido F, Salas A, Torre-Cisneros J, Torres E, et al. Treatment of visceral leishmaniasis in HIV-infected patients: a randomized trial comparing meglumine antimoniate with amphotericin B. Spanish HIV-Leishmania Study Group. AIDS 1999;13:1063-9.

15. Alvar J, Aparicio P, Aseffa A, den Boer M, Canavate C, Dedet JP, et al. The relationship between leishmaniasis and AIDS: the second 10 years. Clin Microbiol Rev 2008;21:334-59, table of contents.

16. Collin S, Davidson R, Ritmeijer K, Keus K, Melaku Y, Kipngetich $S$, et al. Conflict and kala-azar: determinants of adverse outcomes of kala-azar among patients in southern Sudan. Clin Infect Dis 2004;38:612-9.

17. Das VN, Ranjan A, Bimal S, Siddique NA, Pandey K, Kumar N, et al. Magnitude of unresponsiveness to sodium stibogluconate in the treatment of visceral leishmaniasis in Bihar. Natl Med J India 2005;8:131-3.
18. Sundar S, Jha TK, Thakur CP, Engel J, Sindermann H, Fischer C, et al. Oral miltefosine for Indian visceral leishmaniasis. $N$ Engl J Med 2002;347:1739-46.

19. Bhattacharya SK, Sinha PK, Sundar S, Thakur CP, Jha TK, Pandey K, et al. Phase 4 trial of miltefosine for the treatment of Indian visceral leishmaniasis. J Infect Dis 2007;196:591-8.

20. Sundar S, Jha TK, Thakur CP, Sinha PK, Bhattacharya SK. Injectable paromomycin for visceral leishmaniasis in India. $N$ Engl J Med 2007;356:2571-81.

21. Alvar J, Croft S, Olliaro P. Chemotherapy in the treatment and control of leishmaniasis. Adv Parasitol 2006;61:223-74.

22. Respess RA, Rayfield MA, Dondero TJ. Laboratory testing and rapid HIV assays: applications for HIV surveillance in hard-to-reach populations. AIDS 2001;15(Suppl 3):S49-59.

23. Chulay JD, Bryceson AD. Quantitation of amastigotes of Leishmania donovani in smears of splenic aspirates from patients with visceral leishmaniasis. Am J Trop Med Hyg 1983;32:475-9.

24. Thakur CP. Socio-economics of visceral leishmaniasis in Bihar (India). Trans R Soc Trop Med Hyg 2000;94:156-7.

25. Rijal S, Koirala S, Van der Stuyft P, Boelaert M. The economic burden of visceral leishmaniasis for households in Nepal. Trans $R$ Soc Trop Med Hyg 2006;100:838-41.

26. Bhattacharya SK, Sur D, Sinha PK, Karbwang J. Elimination of leishmaniasis (kala-azar) from the Indian subcontinent is technically feasible \& operationally achievable. Indian J Med Res 2006;123:1956.

27. Boelaert M, Criel B, Leeuwenburg J, Van Damme W, Le Ray D, Van der Stuyft P. Visceral leishmaniasis control: a public health perspective. Trans R Soc Trop Med Hyg 2000;94:465-71.

28. Sundar S, Murray HW. Availability of miltefosine for the treatment of kala-azar in India. Bull World Health Organ 2005;83:394-5.

29. Sundar S, Rai M, Chakravarty J, Agarwal D, Agrawal N, Vaillant M, et al. New treatment approach in Indian visceral leishmaniasis: single-dose liposomal amphotericin B followed by short-course oral miltefosine. Clin Infect Dis 2008;47:1000-6.

30. Desjeux P. Leishmaniasis: current situation and new perspectives. Comp Immunol Microbiol Infect Dis 2004;27:305-18. 